\title{
Evaluación de las múltiples desventajas de las niñas mayas: efectos del género, el origen étnico, la pobreza y el lugar de residencia sobre la educación en Guatemala
}

Kelly Hallman

Population Council

Sara Peracca

Jennifer Catino

Population Council

Marta Julia Ruiz

Follow this and additional works at: https://knowledgecommons.popcouncil.org/departments_sbsr-pgy

Part of the Demography, Population, and Ecology Commons, Education Commons, Family, Life Course, and Society Commons, Gender and Sexuality Commons, and the International Public Health Commons How does access to this work benefit you? Let us know!

\section{Recommended Citation}

Hallman, Kelly, Sara Peracca, Jennifer Catino, and Marta Julia Ruiz. 2007. "Evaluación de las múltiples desventajas de las niñas mayas: efectos del género, el origen étnico, la pobreza y el lugar de residencia sobre la educación en Guatemala," Promoción de transiciones a la vida adulta sanas, seguras y productivas Resumen no. 16. New York: Population Council. 


\section{Evaluación de las múltiples desventajas de las niñas mayas: efectos del género, el origen étnico, la pobreza y el lugar de residencia sobre la educación en Guatemala}

\section{Preparado por Kelly Hallman, Sara Peracca, Jennifer Catino y Marta Julia Ruiz}

E I acceso a la educación primaria en Guatemala se incrementó en los últimos años, especialmente en las áreas rurales (Anderson 2001). No obstante, los índices de finalización de escuela primaria y de alfabetismo correspondientes a personas jóvenes continúan estando entre los más bajos de Latinoamérica, y persisten otros problemas, como por ejemplo ingreso tardío, repetición de cursos y abandono temprano (UNESCO 2003 y 2006). Se estima que el índice de alfabetismo entre adultos es del $85 \%$ en Latinoamérica en general, comparado con sólo un $70 \%$ en Guatemala (UNDP 2004). Aunque los pueblos indígenas de Latinoamérica por lo general tienen menos escolaridad que los no indígenas, las diferencias étnicas son mayores en Guatemala, donde los adultos indígenas (mayas) tienen menos de la mitad del nivel de escolaridad que los adultos no indígenas (ladinos): 2.5 años comparados con 5.7 años (Hall y Patrinos 2005). Las tendencias recientes muestran que el vacío étnico se está reduciendo entre los jóvenes; sin embargo, sigue habiendo grandes desigualdades. Entre los jóvenes de 10 a 19 años de edad, el índice de alfabetismo maya equivale a cuatro quintos de la tasa ladina: el $74 \%$ comparado con el 90\% (Shapiro 2005).

En Guatemala también son grandes las diferencias de género en cuanto a alfabetismo y educación. La relación femenina-masculina de alfabetismo es 0.77 entre los adultos y 0.86 en los jóvenes de 15 a 24 años de edad. Además, aunque la relación de matriculación en la escuela primaria entre niñas y niños, correspondiente a 0.95 en 2000 , indica grandes mejoras en los índices de ingreso escolar, la relación de finalización de escuela primaria entre mujeres y varones de 15 a 24 años de edad es sustancialmente menor: 0.82 (ENCOVI 2000).

Las mujeres mayas constituyen, sin duda, el grupo más desfavorecido. Sólo el 39\% de las mujeres mayas de 15 a 64 años de edad en Guatemala están alfabetizadas (comparado con el 68\%, $77 \%$ y $87 \%$ de los varones mayas, las mujeres ladinas y los hombres ladinos respectivamente), y sólo dos tercios de las mujeres mayas de 10 a 19 años de edad están alfabetizadas (en com-

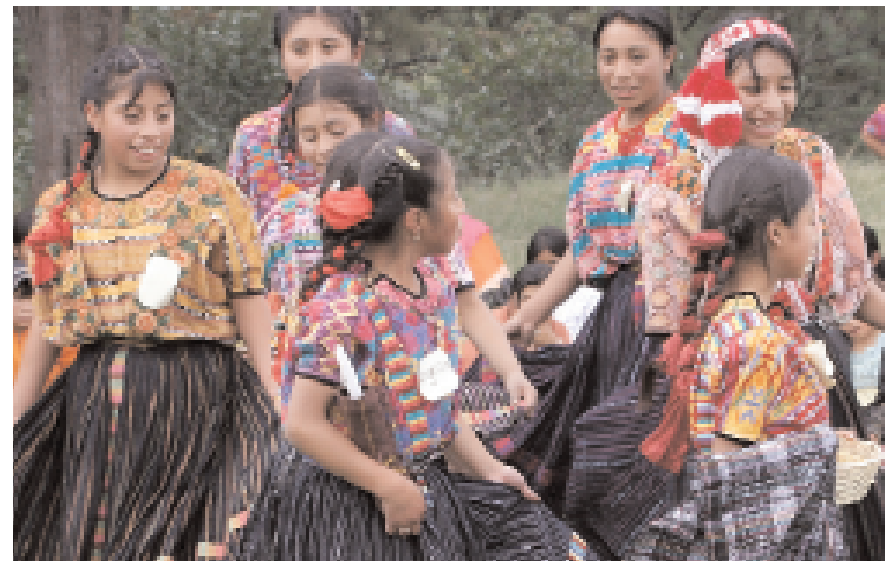

paración con el $80 \%$ de los hombres mayas y el $90 \%$ de las mujeres y hombres ladinos) (Shapiro 2005).

Los mayas representan el $42 \%$ de la población de Guatemala, habitan principalmente en áreas rurales y políticamente están poco representados. Tres cuartos de los mayas son pobres, comparados con el $40 \%$ de los ladinos (ENCOVI 2000).

\section{Matriculación de niñas mayas}

Los datos de la Encuesta Nacional sobre Condiciones de Vida, ENCOVI, correspondiente al año 2000 nos permite estudiar los factores determinantes de matriculación escolar, progresión de grados y logros educativos entre personas de 7 a 24 años de edad. El análisis comienza con niños de siete años de edad, la edad obligatoria de inscripción en la escuela primaria en Guatemala. Hacia los 24 años de edad, la mayoría de los guatemaltecos han finalizado la escuela; por ello, 24 años es el límite de edad superior en el análisis. Una sección detallada de la encuesta referida a consumos y gastos permite calcular los niveles de pobreza a nivel nacional. El Instituto Nacional de Estadísticas reunió los datos de ENCOVI entre 1999 y 2000 de 
una muestra representativa a nivel nacional de 11,170 hogares, 3,544 urbanos y 7,626 rurales.

La Figura 1 muestra el estado de inscripción actual por origen étnico, sexo y edad. Con cada año que pasa, las niñas mayas tienen menos posibilidades de ser inscritas. A los siete años de edad, sólo el $54 \%$ de las niñas mayas permanecen en la escuela, comparado con un $71 \%$ de niños mayas y un $75 \%$ de niñas ladinas. En los cuatro grupos de género-origen étnico, los niveles de matriculación son más altos entre los 9 y los 11 años de edad, y a los 12 años de edad se produce un descenso marcado. Dicha caída es más abrupta en el caso de las niñas mayas: a los 16 años de edad sólo el 25 por ciento de las niñas mayas están inscritas, comparado con aproximadamente la mitad de niños mayas y niñas y niños ladinos. La inscripción femenina maya en las cohortes más jóvenes está creciendo desde el punto de vista absoluto y relativo; sin embargo, la brecha entre género y origen étnico es más pequeña para los niños de 12 años de edad y menores que para los adolescentes.

No obstante, las niñas mayas no constituyen un grupo homogéneo. Los datos de ENCOVI revelan que un cuarto de las niñas mayas clasificadas como no pobres tienen índices de matriculación en la escuela primaria y niveles de grado por edad iguales a los de las niñas ladinas, y entre las que finalizan la escuela primaria, niveles de inscripción en la escuela secundaria que equivalen aproximadamente al $80 \%$ de los de las niñas ladinas. Por otra parte, un cuarto de las niñas mayas clasificadas como extremadamente pobres tienen los peores resultados educativos: sólo la mitad de esas niñas en edad de escuela primaria ingresaron a la escuela, menos del 10\%de las niñas de 13 a 24 años que ingresaron a la escuela primaria completaron ese nivel, y sólo un $14 \%$ de estos graduados de escuela primaria se inscribieron alguna vez en la escuela secundaria. La Figura 2 muestra la proporción de las niñas mayas inscritas en la escuela según la posición económica.

\section{Las niñas mayas en la escuela primaria}

Como ya mencionamos anteriormente, los índices de matriculación escolar de los niños guatemaltecos caen abruptamente a los 12 años de edad. Si bien esa edad marca una transición entre la educación

Figura 1. Proporción de mayas y ladinos actualmente inscritos en la escuela primaria o secundaria, por edad

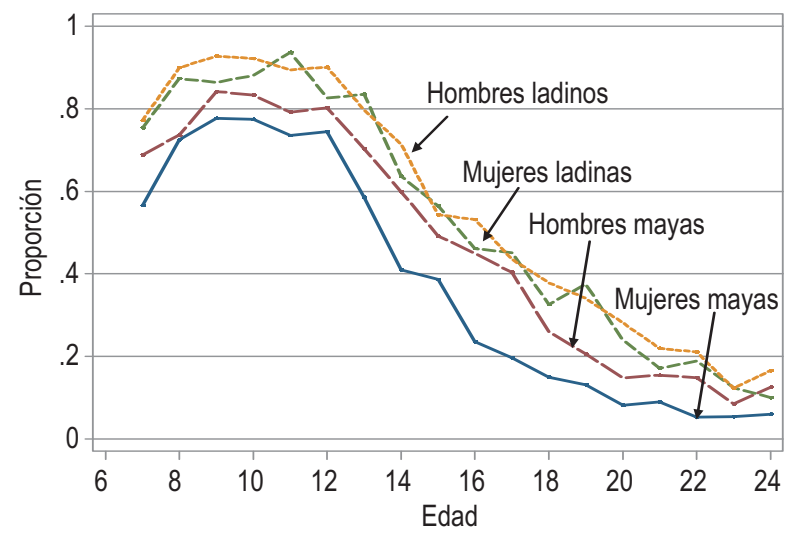

primaria y secundaria para los niños que ingresaron a la escuela a los siete años de edad y tuvieron un buen progreso, la mayoría de los niños de 12 años y mayores, especialmente mayas, alcanzan logros muy bajos y pocos han completado la escuela primaria. Aunque en Guatemala es obligatorio asistir a la escuela a partir de los siete años de edad, no todos los niños se inscriben a esta edad. La decisión de los padres con respecto a cuándo inscribir a su hijo (y si inscribirlo) en la escuela tiene importantes consecuencias para el progreso educativo futuro del niño y sus logros. Según los datos de ENCOVI, los niños mayas comienzan la escuela medio año más tarde que los niños ladinos. En el caso de las niñas mayas, las diferencias en la edad de inicio escolar según la posición económica son amplias: las que viven en hogares extremadamente pobres y se matricularon, lo hicieron 0.73 año más tarde que las niñas pertenecientes a hogares medianamente pobres, y 1.2 año después que las niñas con hogares clasificados como no pobres. La edad de ingreso a la escuela primaria para las niñas mayas que no son pobres es aproximadamente la misma que para los niños ladinos.

Junto con la incidencia de inscripción inicial y el momento de inscripción, la continuación escolar (retención) y la repetición de grados constituyen factores básicos que determinan los logros educativos. Se puede obtener una mejor perspectiva del predominio de los estudiantes de más edad examinando el grado por edad, lo cual abarca el comienzo tardío, la repetición de grados y el abandono seguido de reinscripción. Los niños mayas tienen niveles mucho más bajos de grado por edad que los ladinos. Entre los mayas, los niveles de grado por edad correspondientes a las niñas son inferiores a los que corresponden a los niños hasta los 15 años de edad. Sin embargo, a partir de los 16 años, los niveles de grado por edad entre las niñas mayas son superiores a los de los niños mayas; este giro puede indicar que sólo las niñas mayas más calificadas a nivel académico continúan estudiando después de los 15 años. De conformidad con los hallazgos correspondientes a los niveles de matriculación a escuela primaria y edad de ingreso, las niñas mayas que no son pobres tienen niveles de grado por edad casi iguales a los de los estudiantes ladinos. La mayoría de las niñas mayas no pueden finalizar la escuela primaria debido a la pobreza, la carga de trabajo doméstico y las barreras culturales.
Figura 2. Proporción de mujeres mayas actualmente inscritas en la escuela primaria o secundaria, por situación económica

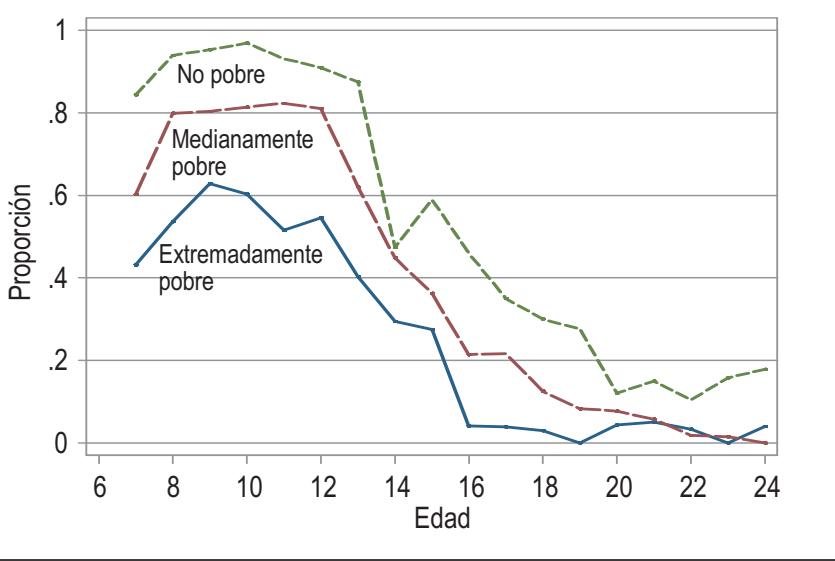


En cuanto a los jóvenes no inscritos al momento de la encuesta, ENCOVI preguntó cuál era la razón principal para la no inscripción. Para los niños en edad de escuela primaria (de 7 a 12 años de edad), la falta de dinero fue el único factor mayor identificado, y su predominio no varió según el género y el origen étnico. La falta de interés en la escuela fue la segunda razón más citada, seguida de la "edad," es decir, por tener más edad de la que corresponde a un grado determinado. Entre las niñas mayas, la edad fue el factor más mencionado entre las extremadamente pobres.

Además de marcar una etapa de la vida en la que los niños comienzan a asumir papeles de trabajo adulto según el género, los 12 años de edad señalan el comienzo de la pubertad, y también el momento en que los padres empiezan a preocuparse de que sus hijas se relacionen con los varones. En un estudio relacionado acerca de los obstáculos y las limitaciones para la educación y la participación social entre niñas y niños mayas del área rural, descubrimos que los padres temían que sus hijas adolescentes interactuaran con los varones, porque consideraban que esto dañaba potencialmente la reputación de sus hijas y sus futuras perspectivas matrimoniales; también opinaban que sus hijas corrían un riesgo mayor de quedar embarazadas antes de casarse (Colom y otros, 2004). (Los nacimientos fuera del matrimonio o la unión consensual no son comunes y son altamente estigmatizados en las comunidades mayas rurales.)

Entre las persona encuestadas de 13 a 24 años de edad, las razones más mencionadas para no matricularse en la escuela fueron las tareas de la casa (para las mujeres) y el trabajo (en el caso de los varones). Entre las mujeres ladinas fue más probable que entre las mujeres mayas el hecho de mencionar el trabajo en el mercado como razón para no matricularse, en comparación con las tareas de la casa. Entre ambos sexos, la falta de dinero fue la segunda razón más común, con pocas diferencias por origen étnico. Entre las mujeres mayas no inscritas, las respuestas variaron según la situación económica. Fue mucho más probable que las de condición pobre mencionaran la falta de dinero y el trabajo de la casa, mientras que otras mencionaron principalmente el trabajo y la falta de interés.

Generalmente, en los países en desarrollo, el matrimonio antes de los 18 años de edad se vincula, en el caso de las mujeres, a índices más bajos de inscripción escolar y logros educativos (Mensch 2005). La edad al matrimonio en Guatemala es menor para las mujeres mayas que para las ladinas, y las diferencias étnicas comienzan a aparecer aproximadamente a los 15 años de edad. Hacia los 18 años de edad, casi el $40 \%$ de las mujeres mayas están casadas; casi el doble del porcentaje de mujeres ladinas de la misma edad. A pesar de la edad temprana de matrimonio, existe una brecha entre la edad media de abandono de la escuela y la edad de matrimonio de las niñas mayas. Asimismo, debido a que la mayor parte de los embarazos en Guatemala se producen en el marco del matrimonio, el embarazo de mujeres solteras no es una causa probable de abandono escolar. Aunque el momento en que las niñas abandonan la escuela no coincide directamente con el matrimonio, es posible que las expectativas de los padres con respecto a la vida futura de sus hijas influyan en lo que invierten para su educación. Nuestro trabajo cualitativo en estas comunidades (Colom y otros, 2004) reveló que los padres se mostraban reacios a invertir en la educación de sus hijas más allá de la pubertad, debido a los altos costos directos y de oportunidad, y debido a que la mayoría de los padres esperaban que los papeles que sus hijas desempeñarían en el futuro serían principalmente los de esposa y madre, para los cuales no se consideraba necesaria una educación superior.

\section{Políticas para atraer y mantener a las niñas mayas en la escuela}

Nuestro análisis indica que las mujeres mayas —especialmente las de condición pobre o las que viven en áreas rurales- constituyen el grupo más desfavorecido en Guatemala en cuanto a educación. Es menos probable que estas mujeres se inscriban en la escuela; y cuando lo hacen, es más probable que comiencen tardíamente y que la abandonen antes. Entre los niños de Guatemala que están inscritos, las niñas mayas tienen los niveles más bajos de grado por edad. A pesar de la proporción cada vez mayor de mujeres mayas que participan en el sistema educativo, es común que las jóvenes guatemaltecas abandonen la escuela, especialmente a los 12 años; a esta edad, es especialmente frecuente el abandono escolar entre las mujeres mayas. A pesar de que el porcentaje de hombres y mujeres mayas inscritos es aproximadamente el mismo a los diez años de edad (alrededor de un $80 \%$ ) según ENCOVI, sólo el $60 \%$ de los varones mayas y el $40 \%$ de las niñas mayas continúan en la escuela a los 14 años de edad.

Los 12 años de edad marcan una transición entre los niveles de primaria y secundaria para aquellos niños que ingresaron en la escuela a la edad requerida y tuvieron un progreso regular. Sin embargo, la mayoría de los niños no inscritos de 12 a 18 años de edad tienen un nivel de logros bajo, y pocos completaron la escuela primaria. Además de los programas de reducción de la pobreza, es posible que los esfuerzos del gobierno para alentar a las familias a comenzar la educación de sus hijos a los siete años de edad conduzcan menos intereses en conflicto con respecto a la asignación de tiempo a medida que los niños se acercan a la pubertad y son obligados a asumir responsabilidades de adultos.

La razón principal mencionada por todos los niños guatemaltecos en edad de escuela primaria para no estar actualmente inscritos fue la falta de dinero. La "edad" (tener más edad que la que corresponde a un grado determinado) y la falta de interés fueron la segunda y tercera razones más comunes. Estos hallazgos apuntan a la necesidad de difundir más ampliamente becas y otros programas de incentivo educativo. Si bien el enfoque del gobierno con respecto a la concentración en las áreas rurales es positivo, quizá no sea suficientemente preciso. Aunque los hogares con pobreza extrema están situados de manera desproporcionada en las áreas rurales, una cuarta parte de los hogares rurales encuestados en ENCOVI no eran pobres.

Algunos estudios anteriores han demostrado que aumentar el acceso a los programas de educación bilingüe en los primeros grados disminuye la repetición de grados y el abandono entre los 
estudiantes mayas de escuela primaria (Morren 1988; Patrinos y Velez 1996; Enge y Chesterfield 1996). Actualmente, sólo una tercera parte de los niños que habitan en zonas rurales en Guatemala tienen acceso a dichos programas (Shapiro 2005). Se ha sugerido que el gobierno ponga en práctica otros programas innovadores-como por ejemplo los utilizados en otros países de Latinoamérica-que permitan a los jóvenes pobres que habitan en zonas rurales asistir a la escuela de maneras no convencionales y culturalmente aceptables, por ejemplo, a través de videoconferencias y cursos por correspondencia. Nuestra investigación cualitativa en comunidades rurales de las tierras altas (Colom y otros, 2004) reveló que las niñas mayas no inscritas - la mayoría de ellas dedicadas principalmente a las actividades domésticasestán aisladas socialmente; los grupos de la iglesia son la única forma de interacción que la mayoría tiene fuera de sus hogares. Los programas innovadores para estas niñas, que combinan instrucción académica con interacción social en espacios seguros de la comunidad local, pueden ampliar las habilidades de las niñas y expandir sus redes sociales y fuentes de apoyo social (Stromquist y otros, 1999). Es probable que la movilización del apoyo de la comunidad y el trabajo con organizaciones mayas confiables mejoren la aceptación cultural, la eficacia y la durabilidad de dichos programas.

\section{Referencias y publicaciones relacionadas}

Anderson, Maria. 2001. "Guatemala: The education sector," Guatemala Poverty Assessment Program, Technical Paper No. 2. Washington, DC: World Bank.

Catino, Jennifer, Kelly Hallman, and Marta Julia Ruiz. 2007. "Building skills to enhance life opportunities for Mayan girls." Promoting Healthy, Safe, and Productive Transitions to Adulthood Brief No. 5. New York: Population Council.

Colom, Alejandra, Marta Julia Ruiz, Jennifer Catino, Kelly Hallman, Sara Peracca, and Kristen M. Shellenberg. 2004.

"Voices of vulnerable and underserved adolescents in Guatemala," project report prepared for National Youth Forum, Guatemala City, Population Council.

Enge, Kjell I. and Ray Chesterfield. 1996. "Bilingual education and student performance in Guatemala." International Journal of Educational Development 16(3): 291-302.

Hall, Gillette and Harry Anthony Patrinos (eds.). 2005. Indigenous Peoples, Poverty and Human Development in Latin America 1994-2004. Washington, DC: World Bank.
Hallman, Kelly, Sara Peracca, Jennifer Catino, and Marta Julia Ruiz. Forthcoming. "Indigenous Girls in Guatemala: Poverty and Location," in M. Lewis and M. Lockheed (eds.), Exclusion, Gender and Schooling: Case Studies from the Developing World. Washington, DC: Center for Global Development. Instituto Nacional de Estadística. 2000. Encuesta Nacional sobre Condiciones de Vida (ENCOVI). Guatemala: ENCOVI.

Mensch, Barbara. 2005. "The transition to marriage," in Cynthia B. Lloyd (ed.), Growing Up Global: The Changing Transitions to Adulthood in Developing Countries. Washington, DC: National Academies Press, pp. 416-505.

Morren, R. C. 1988. "Bilingual education curriculum in Guatemala," Journal of Multilingual and Multicultural Development 9(4): 353-370.

Patrinos, Harry Anthony and Eduardo Velez. 1996. "Costs and benefits of bilingual education in Guatemala: A partial analysis," Human Capital Development Working Paper 74. Washington, DC: World Bank.

Shapiro, Joseph. 2005. "Guatemala," in Gillette Hall and Harry Anthony Patrinos (eds.), Indigenous Peoples, Poverty and Human Development in Latin America 1994-2004.

Washington, DC: World Bank.

Stromquist, Nelly P., Steven Klees, and Shirley J. Miske. 1999. "Improving girls' education in Guatemala: Impact evaluation," United States Agency for International Development Report PN-ACA-919.

United Nations Development Programme (UNDP). 2004. Human Development Report: 2004. New York: Oxford University Press.

United Nations Educational, Scientific and Cultural Organization (UNESCO). 2003. Achieving the Education Goals. Santiago, Chile: Regional Education Indicators Project.

___ 2006. Global Education Digest 2006: Comparing Education Statistics Across the World. Montreal, Quebec: UNESCO Institute for Statistics.

\section{Donantes}

Center for Global Development, Department for International Development (DFID), The William and Flora Hewlett Foundation, The Andrew W. Mellon Foundation

Para obtener más información o copias de los resúmenes de esta serie, escriba a publications@popcouncil.org Para consultar sobre recursos adicionales, visite www.popcouncil.org/pgy

Population Council

One Dag Hammarskjold Plaza

Nueva York, NY 10017 E.U.A.

(c) 2007 The Population Council, Inc. 